\title{
Evolution of dynamic shear strength of frictional interfaces during rapid normal stress variations
}

\author{
Vito Rubino ${ }^{1 *}$, Yuval Tal ${ }^{2,3}$, Ares J. Rosakis ${ }^{1}$, and Nadia Lapusta ${ }^{2,4}$ \\ ${ }^{1}$ California Institute of Technology, Department of Aerospace (Galcit), 91125 Pasadena (CA), USA \\ ${ }^{2}$ California Institute of Technology, Division of Geological and Planetary Sciences, 91125 Pasadena \\ (CA), USA \\ ${ }^{3}$ Ben-Gurion University of the Negev, Department of Earth and Environmental Sciences, 84105 Beer- \\ Sheva, Israel \\ ${ }^{4}$ California Institute of Technology, Division of Engineering and Applied Science, 91125 Pasadena \\ (CA), USA
}

\begin{abstract}
Pressure shear plate impact tests have revealed that when normal stress changes rapidly enough, the frictional shear resistance is no longer proportional to the normal stress but rather evolves with slip gradually. Motivated by these findings, we focus on characterizing the dynamic shear strength of frictional interfaces subject to rapid variations in normal stress. To study this problem, we use laboratory experiments featuring dynamic shear cracks interacting with a free surface and resulting in pronounced and rapid normal stress variations. As dynamic cracks tend to propagate close to the wave speeds of the material, capturing their behavior poses the metrological challenge of resolving displacements on the order of microns over timescales microseconds. Here we present our novel approach to quantify the full-field behavior of dynamic shear ruptures and the evolution of friction during sudden variations in normal stress, based on ultrahighspeed photography (at 1-2 million frames/sec) combined with digital image correlation. Our measurements allow us to capture the evolution of dynamic shear cracks during these short transients and enable us to decode the nature of dynamic friction.
\end{abstract}

\section{Introduction}

The study of dynamic shear cracks along frictional interfaces is relevant to a wide range of problems, including fiber pull-out in the failure of composite materials, airplane actuators, and earthquakes. Friction formulations typically assume shear resistance to be proportional to normal stress. However, when normal stress changes rapidly, frictional shear resistance no longer obeys proportionality to the normal stress. Instead it evolves with slip gradually. It is important to emphasize the highly dynamic nature of this phenomenon as it occurs over timescales on the order of hundreds of nanoseconds to microseconds. The delayed response

\footnotetext{
* Corresponding author: vito.rubino@,caltech.edu
} 
of the shear resistance to rapid normal stress variations was first observed in experiments performed using the pressure shear plate impact (PSPI) test by Prakash and Clifton [1] (Figure 1). This work was developed in the context of high-speed machining applications and was aimed at better characterizing the frictional response of the interface between tool and workpiece to extend tool life and optimize machining costs. The proper representation of these effects in friction formulations is key for investigations of a wider class of engineering and geophysics problems. Some examples include: (i) slip on locally rough/nonplanar interfaces [2]; (ii) dynamic rupture on bimaterial systems [3-8]; and (iii) the dynamics of ruptures on thrust and normal faults near the Earth's surface, which is important for nearfault shaking and tsunami generation, and which contains rapid changes in the fault-normal stress due to the interaction of rupture with the free surface [9-11]. Several different formulations have been proposed that incorporate this effect [1,3, 12-14]. Ignoring this effect results in ill-posed problems and wrong results of numerical simulations [e.g. 3, 4].

Recently, we have developed an approach based on ultrahigh-speed digital image correlation (DIC) $[15,16]$ that enables us to quantify the full-field behavior of dynamic ruptures [17, 18] and the evolution of dynamic friction [19]. One key feature of our experimental setup is that it is able to produce and record rapid normal stress variations during dynamic rupture next to the intersection of the interface with the side free surface [11], and therefore it is well suited to study this problem. Previous versions of this experimental setup have been successfully used to reproduce a number of dynamic rupture phenomena, including supershear transition, bimaterial effect, and pulse-like rupture propagation [5, 2022]. In this study, we discuss our dynamic measurements and analysis aimed at understanding the evolution of the shear resistance in response to rapid normal stress variations, based on our recent advances [23].

(a)

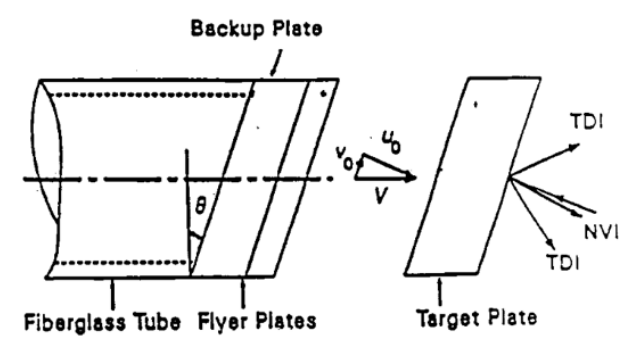

(b)

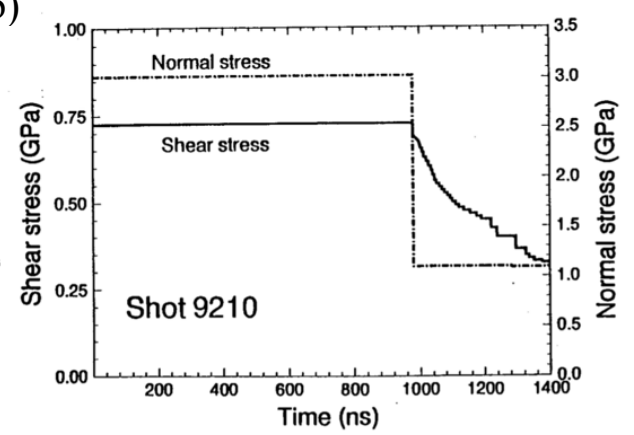

Fig. 1. (a) Experimental setup employed by Prakash and Clifton (1993) [1] to produce sudden normal stress variations. (b) Effect of sudden normal stress change on shear stress which is equal to shear resistance during slip: instead of changing immediately and proportionally, the shear stress experiences gradual evolution. Modified from Prakash and Clifton (1993).

\section{Experimental setup and data processing}

The laboratory setup features a dynamic rupture along the frictional interface, inclined by an angle $\alpha$, formed by two quadrilateral sections of Homalite-100 (Figure 2). The applied compressive load $P$ results in normal and shear prestresses on the interface given by $\sigma_{0}=$ $\cos ^{2} \alpha$ and $\tau_{0}=P \sin \alpha \cos \alpha$, respectively. Ruptures are initiated by the small burst of a Ni$\mathrm{Cr}$ wire placed across the interface. A flash light (Cordin 640) is triggered $200 \mu$ s prior to the wire detonation in order to ramp up in intensity and guarantee adequate illumination during 
the transient. The imaged area of the specimen is covered by a black and white speckle pattern to provide a characteristic pattern for image matching.

\subsection{Full-field measurements with traction continuity across the interface}

Evolving ruptures are captured by an ultrahigh-speed camera (Shimadzu HPV-X) at 1-2 million frames/sec, depending on the experiment, and with an exposure time of $200 \mathrm{~ns}$ (Figure 2). The sequence of images is subsequently analyzed with a local digital image correlation algorithm (VIC-2D, Correlated Solutions Inc.). The analysis is performed independently over the two domains separated by the interface, in order to avoid smoothing of the displacement discontinuity across the interface. Accurate measurements of displacements and stresses near the interface are very important to study the dynamics of shear cracks. However, DIC algorithms involve small errors that can lead to non-physical discontinuities in the stress field across the interface. In order to enforce traction continuity across the interface, we employ an algorithm we have developed to locally adjusts the displacements computed by DIC [24]. The procedure is based on extrapolating the displacements near the interface using polynomials constructed using a constrained inversion, such that (i) traction continuity is satisfied at the interface and (ii) displacements produced by DIC are matched at the center of the pixel where the measurements are accurate. Particle velocity and strain fields are subsequently obtained by temporal and spatial differentiation, respectively, of the displacement fields [15].

\subsection{Capturing the evolution of frictional shear resistance}

Stress maps are computed from strain fields using linear elastic constitutive properties, with the dynamic Young's modulus of Homalite $E=5.3 \mathrm{GPa}$ and Poisson's ratio $v=0.35$ [15]. These measurements provide the stress changes associated with dynamic rupture propagation. The total levels of normal and shear stresses are obtained by summing the stress change to the prestress levels $\sigma_{0}$ and $\tau_{0}$, respectively. Friction time history is computed as the ratio of shear to normal stress along the interface and it is tracked along with that of slip (relative displacement along the interface) and its rate.

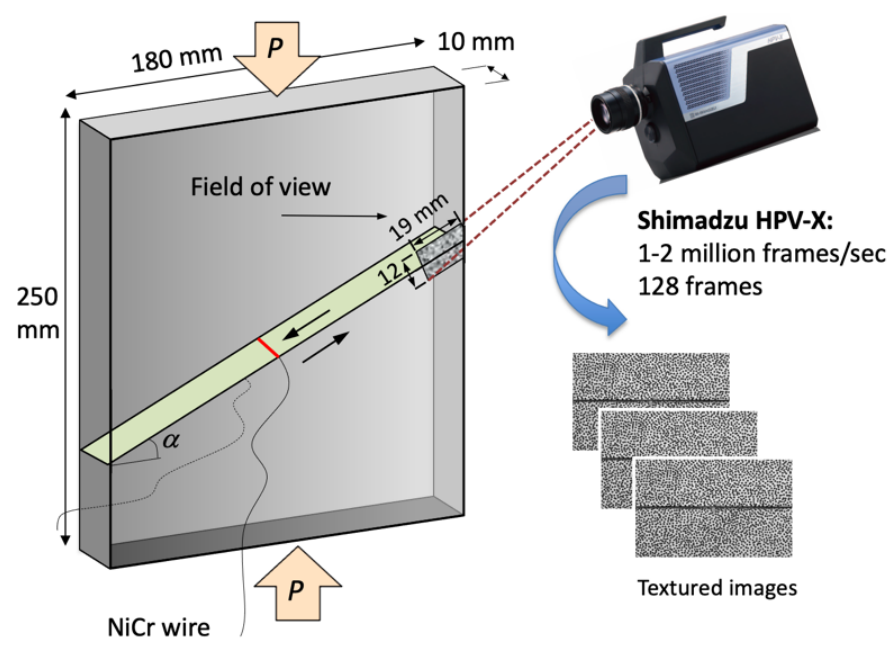

Fig. 2. Schematic of the experimental configuration used in this study featuring a dynamic rupture along a frictional interface approaching the free surface and an ultrahigh-speed camera capturing the transient event at 1 million frames/sec. 


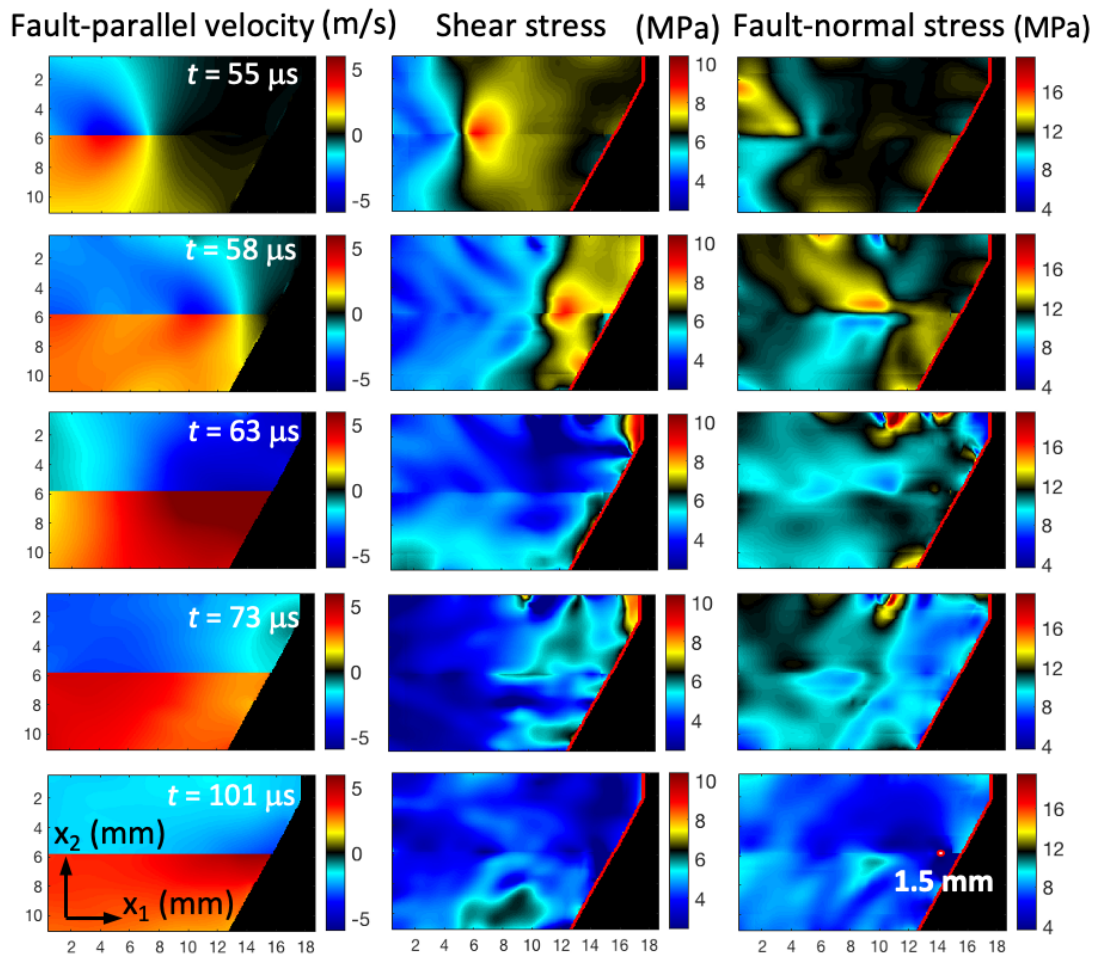

Fig. 3. Full-field of measurements capturing rupture propagation near the free surface with the associated normal stress reduction and shear stress evolution. Full-field interface-parallel velocity (left), shear stress (middle), and interface-normal stress (right) at different stages of a supershear crack-like rupture for a test with $\alpha=29^{\circ}, P=15 \mathrm{MPa}$, and field of view of $18.7 \times 11.7 \mathrm{~mm}$. The first four rows correspond to stages when the rupture arrives to and is reflected from the free surface, while the last row corresponds to the arrival of the trailing Rayleigh rupture.

\section{Dynamic measurements of full-field quantities and friction}

Our recently developed full-field imaging technique $[15,24]$ enables us to image the particle velocities and stress changes within a field of view (FOV) close to the free surface (Figure 3). This approach allows us to both produce full-field maps capturing the dynamics of the phenomenon and to quantify the evolution of interface-normal stress, shear stress, and hence friction along the interface close to the free surface (Figures $4 \mathrm{a}$ and $4 \mathrm{~b}$ ). These measurements allow us to study how friction evolves under the conditions of rapid normal stress variations and to distinguish between different proposed formulations. The full-field interface-parallel velocity, shear stress, and interface-normal stress are shown in Figure 4 at different stages of a supershear crack-like rupture for a test performed with $\alpha=29^{\circ}$ and $P=15 \mathrm{MPa}$, and with a field of view of $18.7 \times 11.7 \mathrm{~mm}$ [23]. The first four rows correspond to stages when the rupture arrives to and is reflected from the free surface, while the last row corresponds to the arrival of the trailing Rayleigh rupture.

Our experimental measurements indicate that the shear resistance does not obey the traditionally assumed proportionality to the normal stress but evolves gradually. This delay is directly observed in plots of the effective friction, $\tau / \sigma$, vs. slip near the free surface (Figure 4) [23]. For experiment 1 in Figure 4, the effective friction initially increases to $\tau / \sigma \sim 0.6$ and 
then decreases with slip to $\tau / \sigma \sim 0.35$ at slip of about $25 \mu \mathrm{m}$. At larger levels of slip, when the impinging rupture is reflected at the free surface, $\sigma$ decreases, and because of the delayed response of the frictional shear resistance $\tau$, the ratio $\tau / \sigma$ increases back to a value of 0.6 at a slip of $120 \mu \mathrm{m}$. The friction $\tau / \sigma$ gradually decreases at larger slip, but as the trailing Rayleigh arrives and $\sigma$ temporarily decreases, $\tau / \sigma$ increases again to a peak of 0.7 , and later drops to 0.4 .

The measurements of $\tau, \sigma$, and $V$ along the interface enable testing different formulations of frictional shear resistance, as well as constraining their parameters. We find that friction formulations without the delayed evolution of shear stress in response to normal stress changes cannot fit our experimental measurements. In model 1 , we test a formulation featuring rate-and-state (RS) friction $[25,26]$ enhanced with flash heating $(\mathrm{FH})$ weakening $[27,28]$ but without accounting for delayed shear stress response to variations in normal stress, so that $\tau=f \sigma$ and the friction coefficient $f$ evolves according to

$$
\begin{aligned}
& f=f_{w}+\frac{\left[f^{*}+a \ln \left(\frac{V}{V^{*}}\right)+b \ln \left(\frac{V^{*} \theta}{L_{R S}}\right)\right]-f_{w}}{1+\frac{L_{R S}}{\theta V_{w}}} \\
& \dot{\theta}=1-\frac{\theta V}{L_{R S}},
\end{aligned}
$$

where $f^{*}$ is the friction coefficient at the reference velocity $V^{*}, a$ and $b$ are RS friction parameters, $L_{R S}$ is the characteristic slip for the state variable evolution, $V_{w}$ is the weakening slip velocity, and $f_{w}$ is the residual friction coefficient. We track the evolution of $\tau / \sigma$ at a point on the interface near the free surface (location marked in Figure 3, bottom right panel) in experiment 1 and find that the friction formulation captures the reduction of $\tau / \sigma$ at slip smaller than $25 \mu \mathrm{m}$. However, at larger slip, as $\sigma$ decreases, the modeled response is significantly below the observed response because the formulation does not account for the delayed response (Figure 4c). In model 2, to account for the effects of rapid normal stress variations, we test a formulation of rate-and-state friction with enhanced-weakening featuring a delayed response of the shear stress according to the Prakash-Clifton law [1, 3, 29]: $\tau=f \psi$, where the function $\psi$ evolves with slip as:

$$
\dot{\psi}=-\frac{V}{L_{P C}}(\psi-\sigma)
$$

where $L_{P C}$ is a characteristic slip scale. This formulation was proposed in the context of regularizing the ill-posed problem of sliding along a bimaterial interface. This model fits the observed frictional response much better than model 1 (Figure 4d). In model 3, we improve the fit with the observed response by considering a formulation of rate-and-state friction with enhanced weakening and Prakash-Clifton law, featuring weakening parameters that depend on normal stress. This formulation is consistent with high-speed friction experiments performed under different normal loads and showed that $V_{w}$ [30] and $f_{w}$ [31] decrease with $\sigma$ in the form of power laws. This formulation does fit better the observed behavior in experiment 1 . Once identified a formulation and its parameters that capture the measurements of experiment 1, we examine its predictive value. Remarkably, we find that the same formulation and parameters allows prediction of the friction evolution near the free surface in various other experiments. A comparison with a selected experiment (labelled as experiment 3) is shown in Figure 4. Accounting for the normal stress dependence of the enhanced-weakening friction parameters $\left(V_{w}\right.$ and $\left.f_{w}\right)$ in the PC law mostly affect experiments performed under lower $\sigma_{0}$ than experiment 1 , and consequently experienced smaller reductions in the friction coefficient. 
(a)

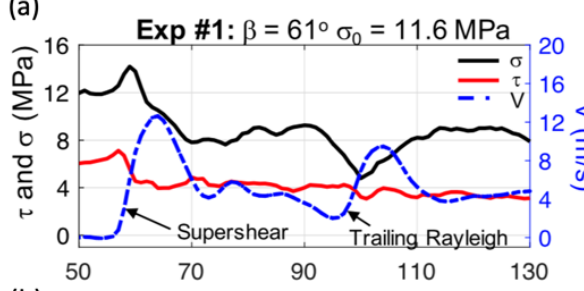

(b)

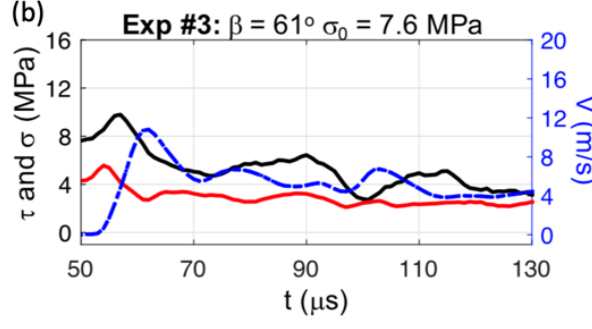

(c)

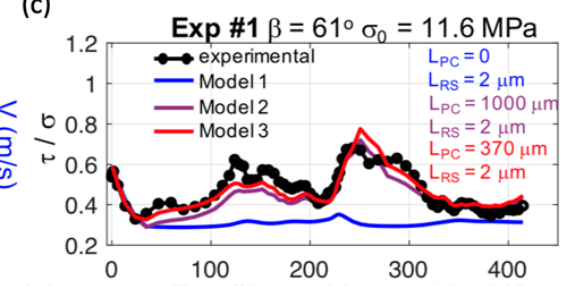

(d)

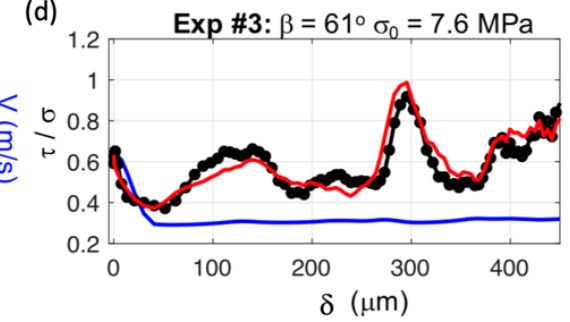

Fig. 4. Experimental measurements showing the delayed response of the frictional shear resistance to rapid normal stress variations and fitting with friction models that capture this phenomenon (modified after Tal et al., 2020). (a-b) Time histories of $\tau$ (red), $\sigma$ (black), and $V$ (blue) near the free surface (for the location marked in the bottom right panel of Figure 3) for experiments labelled as 1 and 3. (c) Fitting of the measured effective friction $\tau / \sigma$ (experiment 1 ) for three models discussed in the text. The experimental data are best fit by friction model 3 with the Prakash-Clifton evolution distance that is two to three orders of magnitude larger than that of rate-and-state friction. (d) Comparison of the measured and predicted values of $\tau / \sigma$ for experiment 3 and friction model 3 . The parameters of the friction model, which have been constrained using the data of experiment 1 , coupled with the measured velocity time history of experiment 3 , allow us to predict the nontrivial friction evolution in this experiment, as well as for various other experiments not show here.

\section{Conclusions}

In this study we have shown the behavior of dynamic shear cracks approaching and interacting with a free surface, using ultrahigh-speed photography coupled with digital image correlation. The full-field measurements show in vivid detail the particle motion characterizing the rupture dynamics and the associated rapid normal stress reductions, occurring over timescales of microseconds. Our findings clearly demonstrate a significant delay between normal stress changes and the corresponding changes in the shear resistance. These conclusions, based on a laboratory configuration featuring the interaction of a shear rupture with the free surface, have important implications for the dynamics of thrust earthquakes near the free surface. In particular, our results indicate that the delay in shear resistance response to variations in normal stress is associated with an evolution distance that is two to three orders of magnitude larger than that of rate-and-state friction. Such delay is also important in many engineering and geophysics problems that involve rapid normal stress variations, such as slip on nonplanar interfaces and bimaterial systems.

We acknowledge the following sources of support: NSF (Grant EAR-1651235), US Geological Survey (USGS) (Grant G20AP00037), Caltech/ Mechanical and Civil Engineering Big Idea Fund (2019), Caltech's Division of Geological and Planetary Sciences, and the Southern California Earthquake Center (SCEC) (Contribution 19093). SCEC is funded by NSF Cooperative Agreement EAR-1033462 and USGS Cooperative Agreement G12AC20038. 


\section{References}

1. V. Prakash and R.J. Clifton, ASME Applied Mechanics Division-publicationsAMD 165, 33-33 (1993).

2. K. Duru and E.M. Dunham, J. Comput. Phys. 305, 185-207 (2016).

3. A. Cochard and J. Rice, J. Geophys. Res. Solid Earth 105, 25891-25907 (2000).

4. J.R. Rice, N. Lapusta, and K. Ranjith, J. Mech. Phys. Solids 49, 1865-1898 (2001).

5. K. Xia, A.J. Rosakis, H. Kanamori, and J.R. Rice, Science 308, 681-4 (2005).

6. Z. Shi, Y. Ben-Zion, and A. Needleman, J. Mech. Phys. Solids 56, 5-24 (2008).

7. H.S. Bhat, R.L. Biegel, A.J. Rosakis, and C.G. Sammis, Tectonophysics 493, 263271 (2010).

8. H. Shlomai and J. Fineberg, Nature communications 7, 1-7 (2016).

9. Oglesby, Archuleta, and Nielsen, Science (New York, N.Y.) 280, 1055-9 (1998).

10. J.E. Kozdon and E.M. Dunham, Earth Planet. Sci. Lett. 396, 56-65 (2014).

11. V. Gabuchian, A.J. Rosakis, H.S. Bhat, R. Madariaga, and H. Kanamori, Nature 545, 336 (2017).

12. M.F. Linker and J.H. Dieterich, J. Geophys. Res. Solid Earth 97, 4923 (1992).

13. G. Povirk and A. Needleman, J. Eng. Mater. T ASME 115, 286-291 (1993).

14. D. Coker, G. Lykotrafitis, A. Needleman, and A. Rosakis, J. Mech. Phys. Solids 53, 884-922 (2005).

15. V. Rubino, A. Rosakis, and N. Lapusta, Exp. Mech. 59, 551-582 (2019).

16. A. Rosakis, V. Rubino, and N. Lapusta, J. Appl. Mech. 87, (2020).

17. M. Gori, V. Rubino, A. Rosakis, and N. Lapusta, Nature Communications 9, 4754 (2018).

18. V. Rubino, A. Rosakis, and N. Lapusta, J. Geophys. Res. Solid Earth 125, e2019JB018922 (2020).

19. V. Rubino, A. Rosakis, and N. Lapusta, Nature Communications 8, 15991 (2017).

20. K. Xia, A.J. Rosakis, and H. Kanamori, Science 303, 1859-61 (2004).

21. X. Lu, N. Lapusta, and A.J. Rosakis, Proc. Natl. Acad. Sci. U.S.A. 104, 18931-6 (2007).

22. A.J. Rosakis, K. Xia, G. Lykotrafitis, and H. Kanamori, Dynamic shear rupture in frictional interfaces-speeds, directionality, and modes, in Treatise on Geophysics. 2007, Elsevier. p. 153-192.

23. Y. Tal, V. Rubino, A.J. Rosakis, and N. Lapusta, Proc. Natl. Acad. Sci. U.S.A. 117, 21095-21100 (2020).

24. Y. Tal, V. Rubino, A.J. Rosakis, and N. Lapusta, Appl. Sci. 9, 1625 (2019).

25. J.H. Dieterich, J. Geophys. Res. Solid Earth 84, 2161-2168 (1979).

26. A. Ruina, J. Geophys. Res. Solid Earth 88, 10359-10370 (1983).

27. J.R. Rice, J. Geophys. Res. Solid Earth 111, B05311 (2006).

28. D.L. Goldsby and T.E. Tullis, Science 334, 216-8 (2011).

29. V. Prakash, (1998).

30. K.M. Brown and Y. Fialko, Nature 488, 638-641 (2012).

31. A. Niemeijer, G. Di Toro, S. Nielsen, and F. Di Felice, J. Geophys. Res. Solid Earth 116, (2011). 